\title{
Uso de imagens de sensoriamento remoto para estratificação do cerrado em inventários florestais
}

Sérgio Teixeira da Silva ${ }^{1}$, José Marcio de Mello ${ }^{1}$, Fausto Weimar Acerbi Junior ${ }^{1}$, Aliny Aparecida dos Reis ${ }^{1}$, Marcel Régis Raimundo ${ }^{1}$, lasmim Louriene Gouveia Silva ${ }^{1}$, José Roberto Soares Scolforo ${ }^{1}$

${ }^{1}$ Universidade Federal de Lavras, Departamento de Ciências Florestais, CP 3037, CEP 37.200-000, Lavras, MG, Brasil

*Autor correspondente: alinyreis@hotmail.com

Termos para indexação: Amostragem sistemática Amostragem casual estratificada Interpretação visual de imagens

Sensoriamento remoto

Index terms:

Systematic sampling

Stratified random sampling

Visual image interpretation

Remote Sensing

Histórico do artigo:

Recebido em 13/06/2014

Aprovado em 01/12/2014

Publicado em 31/12/2014

doi: $10.4336 / 2014 . p f b .34 .80 .742$
Resumo - As imagens de sensoriamento remoto podem ser utilizadas como uma ferramenta auxiliar para o inventário de florestas nativas. Nesse sentido, o objetivo deste trabalho foi avaliar o processo de estratificação de um fragmento de cerrado com base em técnicas de interpretação visual de imagens e comparar os erros de estimativa de dois desenhos amostrais, a amostragem casual estratificada (ACE) e a amostragem sistemática (AS). A área de estudo corresponde a um fragmento de cerrado sensu stricto localizado no Município de Papagaios, MG. Os volumes de madeira do cerrado foram obtidos através de um inventário de campo onde 32 unidades amostrais foram medidas sistematicamente. Realizou-se a estratificação da área de estudo utilizando a interpretação visual de uma imagem Landsat $5 \mathrm{TM}$ e os estratos formados foram: "estrato I", "estrato II", “estrato III", água e mata ciliar. Houve redução de $43 \%$ no erro do inventário empregando-se os estimadores da ACE, em relação aos estimadores da AS. Concluiu-se que o procedimento de estratificação baseado em interpretação visual de imagens de sensoriamento remoto foi eficiente, produzindo estratos homogêneos e reduzindo os erros do inventário florestal.

\section{Using remote sensing images for stratification of the cerrado in forest inventories}

\begin{abstract}
Remote sensing imagery can be a very useful auxiliary tool for native forests inventory. Thus, the objective of this study was to evaluate the stratification of a cerrado (Brazilian savanna) patch based on visual image interpretation techniques as well as to compare the errors from two sampling designs, the stratified random sampling (SRS) and the systematic sampling (SS). The study area corresponds to a cerrado sensu stricto patch located in the municipality of Papagaios, Minas Gerais State, Brazil. The cerrado wood volumes were obtained from a forest inventory field campaign where 32 plots were measured systematically. The study area was stratified based on a visual interpretation of a Landsat 5 TM image, and the strata formed were: "strata I", "strata II", "strata III", water and riparian forests. There was a reduction of $43 \%$ on the inventory errors using the SS estimators compared to the inventory errors using the SRS estimators. We concluded that the stratification based on image interpretation techniques was efficient since there was a reduction on the cerrado inventory errors.
\end{abstract}




\section{Introdução}

O cerrado é o segundo maior bioma brasileiro, cobrindo aproximadamente $21 \%$ do território nacional. Segundo Neri et al. (2007), o cerrado é o bioma de maior representatividade no estado de Minas Gerais. É responsável por um terço da biodiversidade nacional e por 5\% das espécies mundiais, destacando-se em número de espécies da fauna e da flora (Myers et al., 2000; Klink \& Machado, 2005).

Em decorrência da ampla faixa latitudinal de ocorrência do cerrado, este bioma está sujeito a uma variedade de fatores que influenciam sua produtividade, como as condições de solo, clima e fisiografia, dentre outros fatores que afetam diretamente o crescimento, a mortalidade, a densidade e a variabilidade de espécies, resultando assim em um bioma altamente complexo (Ratter et al., 1997).

Durante a realização do Inventário Florestal de Minas Gerais, foram amostrados diversos fragmentos do bioma cerrado, distribuídos nas fitofisionomias campo cerrado, cerrado sensu stricto e cerradão (Scolforo et al., 2008). Os autores utilizaram o procedimento de amostragem em dois estágios, e encontraram uma volumetria média de $18,56 \mathrm{~m}^{3} \mathrm{ha}^{-1}$ para os fragmentos de campo cerrado, $49,97 \mathrm{~m}^{3} \mathrm{ha}^{-1}$ para os fragmentos de cerrado sensu stricto e $117,49 \mathrm{~m}^{3} \mathrm{ha}^{-1}$ para os fragmentos de cerradão. Estes resultados evidenciam que o bioma cerrado no estado de Minas Gerais apresenta indivíduos de porte arbóreo, os quais podem ser utilizados para fins madeireiros desde que estejam dentro da legalidade ambiental.

A obtenção de informações quali-quantitativas das áreas de cerrado exige a adoção de uma série de critérios e metodologias técnicas para que o inventário florestal seja representativo e forneça resultados consistentes e confiáveis sobre os parâmetros estimados (Alvarenga et al., 2012).

Segundo Mello \& Scolforo (2000), em áreas que apresentam grande heterogeneidade é recomendada a aplicação do procedimento da Amostragem Casual Estratificada (ACE), que consiste na subdivisão da população em estratos de maior homogeneidade em relação à característica de interesse a ser avaliada com o intuito de melhorar a precisão do inventário florestal (Kanegae Junior et al., 2007; Assis et al., 2009; Alvarenga et al., 2012; Guedes et al., 2012).

No caso de florestas nativas, a utilização da estratificação esbarra muitas vezes na dificuldade de definição de uma base para a estratificação que represente o comportamento da variável de interesse, bem como a delimitação e a definição das áreas dos estratos (Alvarenga, 2012).

O emprego de técnicas de interpretação visual de imagens provenientes de sensoriamento remoto possibilita a identificação de regiões de maior homogeneidade dentro da área de estudo de acordo com critérios pré-estabelecidos, considerando-se para isso semelhanças entre feições e objetos vizinhos (Sousa et al., 2010). Além disso, a utilização de imagens de sensoriamento remoto permite a obtenção de informações sobre grandes áreas de formações vegetais nativas em um tempo reduzido e com menor custo quando comparado com as atividades em campo (Disperati et al., 2007).

A possibilidade de obtenção de informações sobre as áreas de florestas nativas previamente ao levantamento de campo permite ao executor um planejamento eficiente do inventário florestal, propondo uma melhor distribuição espacial das unidades amostrais de acordo com as características da área, permitindo assim melhor estimativa da média e melhor precisão do inventário.

O objetivo deste trabalho foi avaliar o processo de estratificação com base em técnicas de interpretação visual de imagens de sensoriamento remoto da série Landsat 5 TM de um fragmento de cerrado sensu stricto destinado ao inventário florestal e comparar as estimativas da Amostragem Casual Estratificada (ACE) com as estimativas da Amostragem Sistemática (AS).

\section{Material e métodos}

Os dados do presente estudo são provenientes de um fragmento nativo de cerrado sensu stricto, localizado no município de Papagaios, região central do Estado de Minas Gerais, entre as coordenadas geográficas $19^{\circ}$ $23^{\prime} 30^{\prime \prime}-19^{\circ} 25^{\prime} 00^{\prime \prime} \mathrm{S}$ e $44^{\circ} 36^{\prime} 30^{\prime \prime}$ - $44^{\circ} 37^{\prime} 00^{\prime \prime} \mathrm{W}$. A temperatura média anual da região é de $20,93^{\circ} \mathrm{C}$, média anual de precipitação de $1.350 \mathrm{~mm}$, altitude de 717 metros e área aproximada de 354 ha.

Os dados do inventário florestal foram obtidos lançando-se de forma sistemática 32 unidades amostrais retangulares com área de $1.000 \mathrm{~m}^{2}(10 \mathrm{~m}$ x $100 \mathrm{~m})$. Em cada parcela mediu-se a CAP (circunferência a $1,30 \mathrm{~m}$ do solo) e a altura total com uma vara telescópica de todos os indivíduos com circunferência mínima de $15,7 \mathrm{~cm}$. Todas as unidades amostrais foram georreferenciadas 
e as estimativas de volume de madeira foram obtidas aplicando-se as equações volumétricas geradas por fitofisionomia desenvolvidas por Rufini et al. (2010).

Os dados de sensoriamento remoto foram obtidos a partir de uma imagem do satélite Landsat $5 \mathrm{TM}$, na data de passagem de 07/05/2008 correspondente com a data de coleta dos dados em campo durante o mês de junho de 2008, na órbita 218 ponto 073 , e resolução espacial de $30 \mathrm{~m}$. Utilizou-se as bandas TM3, TM4 e TM5, correspondente as regiões espectrais do vermelho, infravermelho próximo e infravermelho médio, respectivamente, para proceder à composição colorida empregada nos procedimentos de interpretação visual da imagem. A seleção dessas bandas se justifica pela composição colorida das bandas TM3, TM4 e TM5 produzirem um alto contraste espectral entre as classes de cobertura do solo (Araújo Filho et al., 2007), permitindo assim melhores distinções entre as diferenças de densidade da vegetação do cerrado.

Realizou-se a correção geométrica da imagem da área de estudo adotando-se como referência imagens TM/Landsat 5 Geo Cover, e em seguida, realizou-se a estratificação do fragmento de cerrado sensu stricto com base na interpretação visual da imagem na composição colorida 453/RGB (Red-Green-Blue). A estratificação baseada na interpretação visual de imagens fundamentase nos princípios de fotointerpretação, onde o intérprete busca delinear áreas com aspecto homogêneo em função da tonalidade dos pixels, associado com observações de textura, com o meio, com as sombras e com a densidade. Os objetos formados foram resultantes de um procedimento de vetorização manual seguido de uma transformação destes vetores para polígonos (Dlugosz et al., 2009).

Os objetos identificados na primeira etapa foram agrupados com base na densidade de cobertura do solo pela vegetação, em três estratos básicos: "estrato I", "estrato II" e "estrato III".

Para avaliar o desempenho da estratificação, utilizouse o processamento do inventário florestal com base nos valores de volume das parcelas coletados em campo. Foram consideradas duas situações diferentes de processamento do inventário para a mesma grade amostral (Figura 2): a) adoção de toda a área como um único estrato, com o processamento do inventário utilizando-se a Amostragem Sistemática (AS) com os estimadores da Amostragem Casual Simples (ACS); b) considerando a estratificação obtida pela interpretação visual da imagem de sensoriamento remoto, com o processamento do inventário efetuado pelos estimadores da Amostragem Casual Estratificada (ACE). Na situação em que uma mesma parcela localizava-se em dois estratos distintos, no processamento do inventário, a mesma foi considerada como pertencente ao estrato que continha mais de $50 \%$ de sua área. A formulação detalhada de ambos os procedimentos pode ser encontrada em Cochran (1977), Thompson (1996) e Péllico Neto \& Brena (1997).

Os resultados provenientes do processamento da amostragem casual estratificada (ACE) tiveram suas estatísticas comparadas aos resultados do processamento pela amostragem sistemática (AS).

Os programas utilizados para as análises foram ENVI (Environment for Visualizing Images) versão 4.8 e o módulo ArcMap do programa ArcGis versão 10.1 (ESRI, 2010).

\section{Resultados e discussão}

O desenvolvimento da estratificação, apoiado nos princípios de interpretação visual de imagens, possibilitou detectar diferentes regiões sobre a área de estudo, isolando uma variedade de objetos com aparências espectrais semelhantes entre os grupos de pixels que os formam (Figura 1).

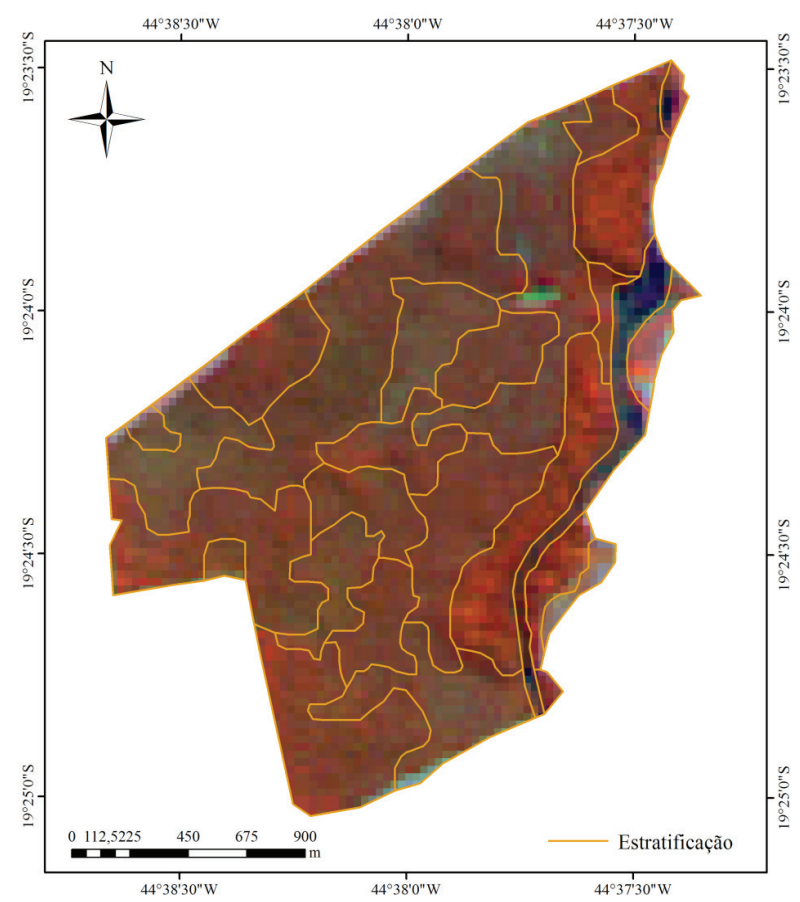

Figura 1. Imagem Landsat $5 \mathrm{TM} 453 / \mathrm{RGB}$ e polígonos resultantes da estratificação. 
Os objetos que apresentaram menor cobertura de vegetação e consequentemente maior exposição do solo foram agrupados e denominados de estrato I, onde se espera obter produtividades e estoques madeireiros menores. Os objetos que apresentaram um recobrimento parcial do solo pela vegetação, sendo possível observar uma mistura de tons indicadores da existência de vegetação e também de partes de solo pouco recoberto foram agrupados e denominados de estrato II. Os objetos que apresentaram maior cobertura do solo pela vegetação de cerrado, indicando uma volumetria de madeira maior, foram agrupados e denominados de estrato III (Figura 2).

Além dos estratos I, II e III foram identificadas outras duas categorias de uso e ocupação do solo, sendo estas a água, correspondente a um rio que atravessa o fragmento e a mata ciliar correspondente a uma fisionomia diferente do cerrado sensu stricto, ajustando-se a um misto de cerrado e mata ciliar devido à proximidade ao rio. As áreas de água e mata ciliar foram desconsideradas durante o processamento do inventário florestal.

$\mathrm{Na}$ Figura 2 encontra-se a estratificação gerada para $o$ fragmento de cerrado em estudo.

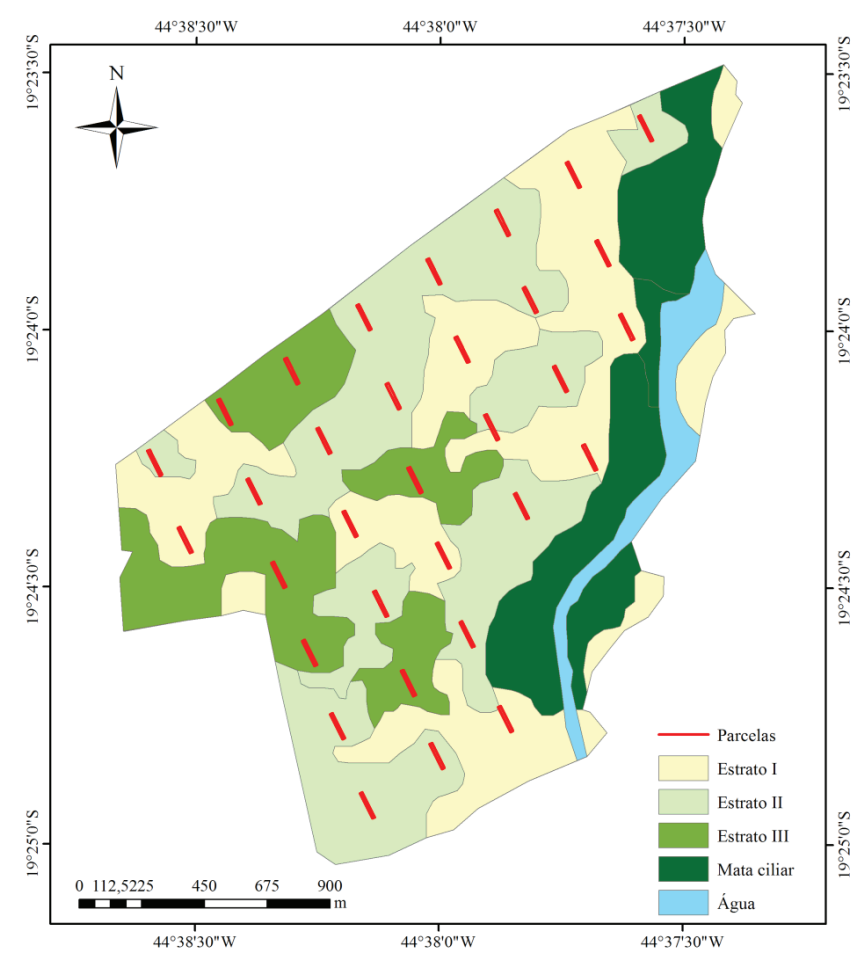

Figura 2. Grade amostral e estratificação obtida a partir da imagem Landsat 5 TM.
Na Tabela 1 encontra-se a quantificação das áreas de cada um dos estratos, bem como as áreas ocupadas por água e mata ciliar. A delimitação dos totais de área por estrato é de fundamental importância para a utilização dos estimadores da amostragem casual estratificada (ACE) no processamento do inventário florestal.

Tabela 1. Áreas dos estratos gerados pela estratificação da imagem Landsat 5 TM para o fragmento de cerrado.

\begin{tabular}{lc}
\hline Estrato & Área do Fragmento (ha) \\
\hline Estrato I & 107,60 \\
Estrato II & 121,20 \\
Estrato III & 59,28 \\
\hline Total com vegetação & $\mathbf{2 8 8 , 0 8}$ \\
de cerrado & 14,56 \\
Água & 51,31 \\
Mata ciliar & $\mathbf{3 5 3 , 9 6}$ \\
\hline Área total &
\end{tabular}

O processamento do inventário florestal foi realizado utilizando-se as 32 unidades amostrais, aplicando-se os procedimentos da amostragem sistemática (AS) e da amostragem casual estratificada (ACE), onde os estratos foram definidos pela interpretação visual da imagem Landsat 5 TM. Os resultados do processamento do inventário, considerando os dois procedimentos de amostragem estão apresentados na Tabela 2.

Tabela 2. Volume médio, desvio padrão, coeficiente de variação $(\mathrm{CV})$, erro do inventário, erro de amostragem e intervalo de confiança para a AS e para a ACE.

\begin{tabular}{lcc}
\hline \multirow{2}{*}{ Estimadores } & \multicolumn{2}{c}{ Procedimento de Amostragem } \\
\cline { 2 - 3 } & AS & ACE \\
\hline $\begin{array}{l}\text { Volume médio } \\
\left(\mathrm{m}^{3} \mathrm{ha}^{-1}\right)\end{array}$ & 101,25 & 99,05 \\
$\begin{array}{l}\text { Desvio padrão } \\
\left(\mathrm{m}^{3} \mathrm{ha}^{-1}\right)\end{array}$ & 26,31 & 14,06 \\
$\mathrm{CV}(\%)$ & 25,98 & 14,20 \\
Erro de amostragem $\left(\mathrm{m}^{3} \mathrm{ha}^{-1}\right)$ & 7,85 & 4,42 \\
Erro de amostragem $(\%)$ & 7,75 & 4,46 \\
$\begin{array}{l}\text { Intervalo de confiança } \\
\left(\mathrm{m}^{3} \text { ha }\right.\end{array}$ & $93,40-109,10$ & $94,63-103,47$ \\
\hline
\end{tabular}

Observa-se que os valores de erro de amostragem percentual de ambos os procedimentos de amostragem ficaram abaixo de $8 \%$, no entanto, deve-se ressaltar que a intensidade amostral nesse fragmento de cerrado foi relativamente alta (1 parcela a cada 10 ha de floresta). 
Alvarenga et al. (2012) utilizaram uma intensidade amostral de 1 parcela a cada 49 ha de cerrado, e encontraram valores de erro de amostragem de $11,38 \%$ para AS e 6,47 para ACE. Os resultados encontrados por Alvarenga et al. (2012) foram superiores aos encontrados nesse estudo, o que pode ser justificado pela menor intensidade amostral adotada por esses autores. No entanto, esses autores também encontraram uma redução significativa do erro do inventário com a utilização da ACE em comparação com a AS.

Aliado à questão da intensidade amostral, tem-se que estas foram distribuídas sistematicamente em toda a área, caracterizando adequadamente a variabilidade espacial da volumetria na área de estudo. Segundo Cressie (1993), a amostragem sistemática capta bem a variabilidade da característica de interesse. Assim, há redução no erro da amostragem em função da melhor representatividade quando se considera a espacialização das parcelas em toda a área (Mello et al., 2009).

No entanto, uma elevada intensidade amostral demanda mais recursos para a realização do inventário florestal. Há uma tendência considerável no setor florestal brasileiro pela busca da minimização do erro reduzindo-se o custo do levantamento florestal e uma das alternativas para alcançar tal objetivo é por meio de técnicas estatísticas que permitam o controle da variabilidade da floresta, que neste caso trata-se do emprego da estratificação (Alvarenga et al., 2012).

Analisando-se a Tabela 2, é possível observar uma redução do coeficiente de variação de $45 \%$ quando se compara a amostragem casual estratificada com a amostragem sistemática, corroborando que, se a base para estratificação adotar critérios pelos quais seja possível reconhecer e isolar áreas homogêneas, o ganho em precisão no levantamento é substancial. Esta redução evidencia que o emprego da interpretação de imagens de sensoriamento remoto para determinação dos estratos foi eficiente, e que a estratificação foi benéfica no sentido de redução do erro de amostragem.

Ainda pela Tabela 2 verifica-se a redução de aproximadamente $44 \%$ no erro de amostragem da amostragem casual estratificada (ACE) em relação à amostragem sistemática (AS). Vale destacar que os intervalos de confiança se sobrepuseram, indicando que as médias estimadas são estatisticamente equivalentes, no entanto, o intervalo da ACE foi mais preciso do que o da AS. Resultados semelhantes puderam ser verificados por Alvarenga (2012) que utilizou a classificação visual de imagens RapidEye de um fragmento de cerrado localizado no município de São Romão, Minas Gerais, para estratificação e posterior análise com os estimadores da ACE.

A redução no coeficiente de variação tem reflexo direto na intensidade amostral. Assim sendo, é possível a utilização de imagens de sensoriamento remoto para estratificação antes do levantamento de campo e para a redução da intensidade amostral (custo do levantamento) sem perda de precisão da estimativa. Outro aspecto importante se refere à representatividade, ou seja, melhor distribuição espacial das parcelas, evitando que ocorram estratos sem amostragem adequada ou a amostragem de áreas impróprias ao manejo florestal.

Espírito Santo et al. (2005) utilizaram de imagens Landsat para a determinação das áreas a serem inventariadas na Floresta Nacional dos Tapajós, bem como para a distribuição dos transectos amostrais em diferentes porções da área de estudo. Esses autores utilizaram a composição 453/RGB para observar diferenças sutis das tipologias vegetais, relacionados aos aspectos da interação da radiação no dossel da vegetação, para facilitar o delineamento amostral na área de estudo.

Vibrans et al. (2010) durante a execução do Inventário Florístico Florestal de Santa Catarina (IFFSC), realizaram a seleção das unidades amostrais a partir de uma estratificação preliminar de floresta e não floresta baseada na interpretação de imagens Landsat e SPOT, corroborando com o potencial da utilização de imagens de sensoriamento como suporte ao inventário florestal de florestas nativas.

Porém, se a avaliação do estoque madeireiro existente visar subsidiar o planejamento das operações de uma empresa, certamente os resultados fornecidos pela ACE trarão maior confiabilidade ao administrador dos recursos florestais. Segundo Guedes et al. (2012) a ACE proporciona um erro de inventário menor do que a amostragem sistemática. Além da redução do erro do inventário, há uma redução na variabilidade da população estratificada, o que implica diretamente na redução da intensidade amostral. Com isso, os custos operacionais da amostragem podem ser reduzidos, uma vez que são fortemente influenciados pelo tempo gasto no processo de demarcação e mensuração das unidades amostrais. 


\section{Conclusões}

As imagens Landsat se mostraram satisfatórias na determinação de estratos destinados ao inventário florestal, permitindo uma boa análise interpretativa da densidade de cobertura vegetal existente na área de interesse.

Os estimadores da amostragem casual estratificada, em termos de precisão, apresentaram-se superiores aos da amostragem sistemática, comprovando a eficácia do processo de estratificação baseado na interpretação visual de imagens do satélite Landsat para geração de estratos com base em imagens de sensoriamento remoto.

Para a realização do inventário em outras áreas de florestas nativas, recomenda-se a avaliação da área a ser inventariada e a identificação de regiões mais homogêneas obtidas a partir do procedimento de estratificação com base nas imagens de sensoriamento remoto. Esse processo poderá apresentar ganhos substanciais em termos de agilidade, custos operacionais e confiabilidade dos resultados obtidos.

\section{Referências}

ALVARENGA, L. H. V. Imagens de alta resolução e geoestatística na estratificação da fisionomia cerrado para inventários florestais. 2012. 92 f. Dissertação (Mestrado em Engenharia Florestal) - Universidade Federal de Lavras, Lavras.

ALVARENGA, L. H. V. Imagens de alta resolução e geoestatística na estratificação da fisionomia cerrado para inventários florestais. 2012. 92 p. Dissertação (Mestrado em Engenharia Florestal) - Universidade Federal de Lavras, Lavras, 2012.

ARAÚJO FILHO, M. C.; MENESES, P. R.; SANO, E. E. Sistema de classificação de uso e cobertura da terra com base na análise de imagens de satélite. Revista Brasileira de Cartografia, Rio de Janeiro, n. 59, v. 02, 2007.

ASSIS, A. L. de.; MELLO, J. M.; GUEDES, I. C. L.; SCOLFORO, J. R. S.; OLIVEIRA, A. D. Development of a sampling strategy for young stands of Eucalyptus sp. using geostatistics. Cerne, Lavras, v. 15 , n. 2, p. 166-173, abr./jun. 2009.

COCHRAN, W. G. Sampling techniques. 3rd. ed. New York: J. Wiley, 1977. $555 \mathrm{p}$.

CRESSIE, A. G. Statistics for spatial data. New York: J. Wiley, 1993. $900 \mathrm{p}$.

DISPERATI, A. A.; SANTOS, J. R.; OLIVEIRA FILHO, P. C.; NEEFF, T. Aplicação da técnica "filtragem de locais máximas" em fotografia aérea digital para a contagem de copas em reflorestamento de Pinus elliottii. Scientia Forestalis, Piracicaba, n. 76, p. 45-55, dez. 2007.
DLUGOSZ, F. L.; ROSOT, N. C.; ROSOT, M. A. D.; OLIVEIRA, Y. M. M. Índice para a avaliação de segmentação de imagens. Floresta, Curitiba, v. 39, n. 1, p. 131-143, 2009.

ESPIRITO-SANTO, F. D. B.; SHIMABUKURO, Y. E.; ARAGÃO, L. E. O. C.; MACHADO, E. L. M. Análise da composição florística e fitossociológica da Floresta Nacional dos Tapajós com o apoio geográfico de imagens de satélites. Acta Amazônica, Manaus, v. 35, n. 2, p. 45-48, 2005.

GUEDES, I. C. de L.; MELLO, J. M.; MELLO, C. R.; OLIVEIRA, A. D.; SILVA, S. T.; SCOLFORO, J. R. S. Técnicas geoestatísticas e interpoladores espaciais na estratificação de povoamentos de Eucalyptus sp. Ciência Florestal, Santa Maria, v. 22, n. 3, p. 541550, jul.-set., 2012.

KANEGAE JÚNIOR, H.; MELLO, J. M.; SCOLFORO, J. R. S; OLIVEIRA, A. D. Avaliação da continuidade espacial de características dendrométricas em diferentes idades de povoamentos clonais de Eucalyptus sp. Revista Árvore, Viçosa, MG, v. 31, n. 5, p. 859-866, set./out. 2007.

KLINK, C. A.; MACHADO, R. B. A conservação do Cerrado brasileiro. Megadiversidade, Belo Horizonte, v. 1, n. 1, p. $147-$ $155,2005$.

MELLO, J. M.; SCOLFORO, J. R. S. Análise comparativa de procedimentos de amostragem em um remanescente de Floresta Estacional Semidecídua Montana. Revista Árvore, Viçosa, MG, v. 24, n. 1, p. 55-62, 2000.

MELlO, J. M. de.; DINIZ, F. S.; OLIVEIRA, A. D.; MELLO, C. R.; SCOLFORO, J. R. S.; ACERBI JUNIOR, F. W. Continuidade espacial para características dendrométricas (número de fustes e volume) em plantios de Eucalyptus grandis. Revista Árvore, Viçosa, MG, v. 33, n. 1, jan./fev., 2009.

MYERS, N.; MITTERMEIER, R. A.; MITTERMEIER, C. G.; FONSECA, G. A. B. da.; KENT, J. Biodiversity hotspots for conservation priorities. Nature, London, v. 403, p. 853-858, 2000.

NERI, A. V.; MEIRA NETO, J. A. A.; SILVA, A. F.; MARTINS, S. V.; SAPORETTI JUNIOR, A. W. Composição florística de uma área de cerrado sensu stricto no município de Senador Modestino Gonçalves, Vale do Jequitinhonha (MG) e análise de similaridade florística de algumas áreas de cerrado em Minas Gerais. Revista Árvore, Viçosa, MG, v. 31, n. 6, p. 1109-1119, 2007.

PÉLLICO NETTO, S.; BRENA, D. A. Inventário florestal. Curitiba, 1997. $316 \mathrm{p}$

RATTER, J. A.; RIBEIRO, J. F.; BRIDGEWATER, S. The Brazilian cerrado vegetation and threats to its biodiversity. Annals of Botany, Oxford, v. 80, p. 223-230, 1997.

RUFINI, A. L.; SCOLFORO, J. R. S.; OLIVEIRA, A. D.; MELLO, J. M. Equações volumétricas para o cerrado sensu stricto em Minas Gerais. Cerne, Lavras, v. 16, n. 1, p. 1-11, jan./mar. 2010.

SCOLFORO, J. R. S.; MELlo, J. M. de; OLIVEIRA, A. D. Inventário florestal de Minas Gerais: Cerrado - florística, diversidade, similaridade, distribuição diamétrica e de altura, volumetria, tendências de crescimento e áreas aptas para manejo florestal. Lavras: UFLA, 2008. 216 p. 
VIBRANS, A. C.; SEVGNANI, L.; LINGNER, D. V.; GASPER, A. L.; SABBAGH, S. Inventário florístico florestal de Santa Catarina (IFFSC): aspectos metodológicos e operacionais. Pesquisa Florestal Brasileira, Colombo, v. 30, n. 64, p. 291-302, 2010. DOI: 10.4336/2010.pfb.64.291
SOUSA, A. M. O.; MESQUITA, P; GONÇALVES, A. C.; SILVA, J. R. M. Segmentação e classificação de tipologias florestais a partir de imagens QUICKBIRD. Ambiência, Guarapuava, v. 6, Ed. Especial, p. 57-66, 2010.

THOMPSON, S. K.; SEBER, G. A. F. Adaptative sampling. New York: Wiley, 1996. 350 p. 
